\title{
Generation of transgenic rodent malaria parasites by transfection of cell culture-derived merozoites
}

\author{
Gesine Kaiser $^{{ }^{*}}$ (D, Mariana De Niz ${ }^{1,3}$, Paul-Christian Burda ${ }^{1,4}$, Livia Niklaus ${ }^{1,2}$, Rebecca Limenitakis Stanway ${ }^{1}$ \\ and Volker Heussler ${ }^{1}$
}

\begin{abstract}
Background: Malaria research is greatly dependent on and has drastically advanced with the possibility of genetically modifying Plasmodium parasites. The commonly used transfection protocol by Janse and colleagues utilizes blood stage-derived Plasmodium berghei schizonts that have been purified from a blood culture by density gradient centrifugation. Naturally, this transfection protocol depends on the availability of suitably infected mice, constituting a time-based variable. In this study, the potential of transfecting liver stage-derived merozoites was explored. In cell culture, upon merozoite development, infected cells detach from the neighbouring cells and can be easily harvested from the cell culture supernatant. This protocol offers robust experimental timing and temporal flexibility.
\end{abstract}

Methods: HeLa cells are infected with P. berghei sporozoites to obtain liver stage-derived merozoites, which are harvested from the cell culture supernatant and are transfected using the Amaxa Nucleofector ${ }^{\circledR}$ electroporation technology.

Results: Using this protocol, wild type P. berghei ANKA strain and marker-free PbmCherry ${ }_{\mathrm{Hsp} 70}$-expressing P. berghei parasites were successfully transfected with DNA constructs designed for integration via single- or double-crossover homologous recombination.

Conclusion: An alternative protocol for Plasmodium transfection is hereby provided, which uses liver stage-derived P. berghei merozoites for transfection. This protocol has the potential to substantially reduce the number of mice used per transfection, as well as to increase the temporal flexibility and robustness of performing transfections, if mosquitoes are routinely present in the laboratory. Transfection of liver stage-derived $P$. berghei parasites should enable generation of transgenic parasites within 8-18 days.

Keywords: Plasmodium berghei, Transfection, Liver stage-derived merozoites

\section{Background}

\section{Genetic manipulation of Plasmodium parasites}

In the past 15 years, Plasmodium parasites have become greatly accessible for genetic manipulation [1-3], facilitated by the genome sequencing of human and rodent malaria parasites [4-6]. Advances continue and Plasmodium knowlesi parasites were recently successfully adapted for in vitro culture in human red blood cells

\footnotetext{
*Correspondence: gesine.kaiser@x100.de

1 Institute of Cell Biology, University of Bern, Bern, Switzerland

Full list of author information is available at the end of the article
}

including successful transfection, which resulted in efficiencies of up to 30\% [7]. Transfection efficiencies of rodent Plasmodium berghei parasites have increased up to $1: 1000$ as a result of implementing the highly efficient non-viral Nucleofector ${ }^{\circledR}$ technology $[1,8]$. A major advantage of using the model organism $P$. berghei for Plasmodium research is the accessibility of the entire life cycle in vitro as well as in vivo, including the liver stage development. A further advantage is the availability of an almost complete genomic DNA library that originated from phage-based vectors, applicable for generation of knock-outs, and tagging of genes [9-11]. Methods for 
typical genetic manipulation, such as the generation of knock-outs and complemented parasites, fluorescent tagging of proteins and even conditional knock-outs, are available for both rodent and human Plasmodium parasites [9, 12-14]. Classically, transfection of DNA constructs into $P$. berghei parasites is performed into blood stage-derived schizonts and merozoites, and benefits from the fact that schizonts do not rupture in in vitro blood cultures and can thus be enriched and purified. Transfection of schizonts and free merozoites, compared to other asexual blood stages, is facilitated by the fact that DNA used for transfection has to cross only two or three membranes, namely the erythrocyte membrane (depending on whether or not merozoites have been released), the parasite plasma membrane (PM) and the nuclear membrane, instead of four, including the parasitophorous vacuole membrane [1]. The standard protocol for $P$. berghei transfection, requires the infection of two mice, which ideally should have a parasitaemia of about $3 \%$ usually achieved between day 5 and 7 after pre-infection. Once the parasitaemia has reached about $3 \%$, blood stage parasites are taken into culture for $16-18 \mathrm{~h}$ and following this, schizonts are purified using a density gradient. Purified schizonts and merozoites are subsequently transfected using the Amaxa Nucleofector ${ }^{\circledR}$ electroporation technology $[1,8]$. This study took advantage of the fact that the merozoite stage of Plasmodium parasites is not restricted to the blood stage, but is also produced at the end of liver stage development. The Plasmodium liver stage is characterized by an immense expansion of the parasite population. Intriguingly, a single sporozoite that has infected a host hepatocyte can mature into thousands of progeny merozoites [15]. At the end of exo-erythrocytic parasite development, merozoites are released from the parasitophorous vacuole (PV) into the hepatocyte cytoplasm. This leads to the detachment of the infected host cell from its neighbouring cells and in in vitro cultures, to detachment of the infected cells, which then float freely in the culture supernatant. Merosomes, sacs containing infectious merozoites, are subsequently extruded from the detached cell and are also found in the cell culture supernatant $[16,17]$. Single detached cells of in vitro-cultured $P$. berghei parasites were recently described to harbour an average of about 4500 individual merozoites $[16,18]$. In a previous study by Stanway et al. individual merosomes or detached cells were collected and used for sub-cloning of transgenic parasites, thereby greatly contributing to the reduction of animals used to achieve clonal transgenic parasite lines [17]. This work presents an established and optimized protocol for transfection of liver stage-derived schizonts and merozoites, which equally aims to reduce the number of animals used for the generation of transgenic parasite lines.

\section{Methods}

\section{Animal work statement}

Experiments were conducted in strict accordance with the guidelines of the Swiss Tierschutzgesetz (TSchG; Animal Rights Laws) and approved by the ethical committee of the University of Bern (Permit Number: BE109/13). Balb/c mice used in experiments were between 6 and 10 weeks of age and were either bred in the central animal facility of the University of Bern, or were supplied from Harlan Laboratories or Charles River.

\section{Culturing of HeLa cells}

HeLa cells (a gift from Robert Menard, Pasteur Institute, Paris) were grown in MEM (minimum essential medium) with Earle's salts (Bioconcept, cat. no 1-31F01-I), supplemented with $10 \%$ heat inactivated foetal calf serum-FCS (Bioswisstec, Cat. No. S4150), 1\% penicillin/streptomycin (Bioconcept, Cat. No. 4-01F00-H) and 1\% L-glutamine (Bioconcept, 5-10K00-H) in a humid incubator with 5\% $\mathrm{CO}_{2}$ at $37^{\circ} \mathrm{C}$. Using accutase (Sigma-Aldrich, Cat. No. A6964), cells were passaged twice a week.

\section{Infection of mosquitoes with Plasmodium berghei}

A P. berghei blood stabilate was injected intraperitoneally into a naïve Balb/c mouse (referred to as 'pre-infection mouse'). When this mouse had reached a parasitaemia of $1-4 \%, 50 \mu \mathrm{l}$ of infected blood, diluted with $150 \mu \mathrm{l}$ PBS, were injected intravenously into a mouse (referred to as 'feed mouse') that 3 days prior to that had been intraperitoneally injected with $200 \mu \mathrm{l}$ phenylhydrazine $\left(6 \mathrm{mg} \mathrm{ml}^{-1}\right.$ in PBS) (Sigma-Aldrich, Cat. No. 114715). When the feed mouse, had reached a parasitaemia of at least $7 \%$, usually 3-4 days after infection, with most of the circulating parasites being gametocytes, the mouse was anaesthetized and used to allow about 100-150 female Anopheles stephensi mosquitoes to feed for $1 \mathrm{~h}$. Infected mosquitoes were kept at $20.5{ }^{\circ} \mathrm{C}$ and $80 \%$ humidity and fed with $8 \%$ fructose containing 0.2\% PABA (Sigma-Aldrich, Cat. No. 100536). Infected mosquitoes were dissected to obtain sporozoites for the detached cell transfection protocol between day 16 and 24 post blood feed. 
In vitro infection of HeLa cells with Plasmodium berghei

Infection of HeLa cells with sporozoites required that mosquitoes infected with either $P$. berghei (ANKA strain) wild type parasites or any other selection-marker-free $P$. berghei parasite line were available.

On the day of the infection, the parasites in these mosquitoes were between day 16 to 24 post-blood feed. For one transfection (if more transfections were intended cells were seeded accordingly), HeLa cells were seeded into three wells of a 24-well plate (Greiner Bio-one, Cat. No. 662160) with $2 \times 10^{4}$ cells per well and cultured in complete MEM culture medium. On the following day at about $16.00,3-5$ infected mosquitoes per transfection were anesthetized with chloroform vapour. Anesthetized mosquitoes were briefly dipped into $70 \%$ (vol/vol) ethanol and then transferred to PBS.

For removal of salivary glands, mosquitoes were successively put into $200 \mu \mathrm{l}$ of infection medium [complete MEM culture medium containing $2.5 \mu \mathrm{g} \mathrm{ml}{ }^{-1}$ amphotericin B (Sigma-Aldrich, Cat. No. A2942)] on a slide and the head was carefully removed using surgical forceps. The two sets of each three salivary gland lobes were isolated from the head or mosquito body, and transferred into an Eppendorf tube containing $20 \mu \mathrm{l}$ of infection medium, kept on ice until infection of HeLa cells. Salivary glands were mechanically disrupted using a pestle (Sigma-Aldrich, Cat. No. Z359947) driven by a Cordless motor (Sigma-Aldrich, Cat. No. Z359971), using approximately 10 pulses of $1-2 \mathrm{~s}$. Next, $300 \mu \mathrm{l}$ of infection medium were added and the number of sporozoites was estimated using a Neubauer chamber.

Per anticipated transfection, 50,000 sporozoites were used, contained in a volume of $600 \mu \mathrm{l}$ infection medium. After removing the medium from the three wells to be infected, $200 \mu \mathrm{l}$ of sporozoite-containing infection medium were added to each of the three wells. To allow fast settling of the sporozoites, the 24-well plate was spun for $1 \mathrm{~min}$ at $200 \mathrm{~g}$. Following $2 \mathrm{~h}$ of incubation time (at $37{ }^{\circ} \mathrm{C}$ with $5 \% \mathrm{CO}_{2}$ ), infected cells were washed with $500 \mu \mathrm{l}$ pre-warmed infection medium to remove mosquito debris and sporozoites that had not been infected. Cells were then incubated with $500 \mu \mathrm{l}$ infection medium in a humid incubator at $37{ }^{\circ} \mathrm{C}$ with $5 \% \mathrm{CO}_{2}$. To avoid fungal and bacterial growth, the infection medium was exchanged at 24 and $48 \mathrm{~h}$ post infection (hpi).

\section{Harvesting detached cells and merosomes}

At about 62-65 hpi (about 09.00 at day 3 post-infection) it was verified by bright-field microscopy that detached cells/merosomes were present in the cell culture supernatant. Detached cells and merosomes are characterized by their round shape. They contain thousands of small merozoites, which, in viable merosomes and detached cells, are constantly moving. If only few detached cells had been formed, infection duration was extended to up to $67 \mathrm{hpi}$. To harvest detached cells and merosomes, the supernatant of each well was gently pipetted up and down three times and finally the medium of the three infected wells was pooled into one Eppendorf tube. Parasites were pelleted at $16,000 \mathrm{~g}$ for $10 \mathrm{~s}$, the direction of the tube was changed by $180^{\circ}$ and the tube was spun again for $10 \mathrm{~s}$ at $16,000 \mathrm{~g}$. Successful centrifugation was verified by the presence of a small semi-transparent/white pellet visible at the bottom of the tube. If more than one transfection was planned, all sets of detached cells were prepared before the transfection was performed.

\section{Transfection of liver stage-derived merozoites}

The transfection procedure was adapted from the previously published protocol by Janse et al. [8]. Mice to be injected with transfected parasites were prewarmed using an infrared lamp for about 5-10 min prior to the injection, to allow the tail vein to dilate. 5-10 $\mu \mathrm{g}$ of DNA (contained in a maximum volume of $10 \mu \mathrm{l})$ were added to $100 \mu \mathrm{l}$ Nucleofector solution 88A6 (Lonza, Cat. No. VVPA-1002 KT) and the same was done for each of the constructs to be transfected. Next, using a $1000-\mu l$ pipette, the supernatant was carefully removed from one detached cell-containing Eppendorf tube, without touching the detached cell pellet. Subsequently, $100 \mu \mathrm{l}$ of DNA-containing Nucleofector solution 88A6 were added and the detached cells and merosomes were cautiously resuspended. Resuspended parasites were transferred from the Eppendorf tube into an electroporation cuvette and placed into the Amaxa Nucleofector device. Parasites were transfected using the program U33 of the Amaxa Nucleofector device. Immediately after transfection, $50 \mu \mathrm{l}$ of RPMI1640 culture medium (Bioconcept, Cat. No. 1-41F01-I) were added to the transfected parasites. Following this, the whole volume of the cuvette (about $150 \mu \mathrm{l})$ was transferred into an Eppendorf tube using the provided Pasteur plastic pipette. Using an insulin syringe, the entire suspension was injected into the tail vein of a naive Balb/c mouse (6-10 weeks of age) and the previous steps were repeated until all constructs were transfected. 


\section{Drug selection and collection of transgenic parasites}

One day after transfection, drugs suitable for selection of transgenic parasites were given to infected mice (depending on the transfected construct), as previously published [8]. Drug selection was continued until transgenic parasites had reached a parasitaemia of $2 \%$ or higher. From 5 days after the transfection onwards, the parasitaemia of mice injected with transfected parasites was checked by thin blood smear. If the transfected construct encoded fluorescent or luminescent markers expressed under a promoter active in the blood stage, fluorescence or luminescence was also checked.

\section{Detection of parasites by fluorescence}

To stain the parasite DNA, a drop of blood from the infected mouse was added to $50 \mu \mathrm{l}$ of PBS containing $1 \mu \mathrm{g} \mathrm{ml}^{-1}$ Hoechst 33342 (Sigma-Aldrich, Cat. No. B2261) in PBS. Parasites were analysed under a fluorescence microscope using the appropriate filters and an increasing magnification of between $200 \times$ and $1000 \times$.

\section{Detection of parasites by luminescence (NanoLuc)}

Parasite detection by luminescence was performed as described before [18]. Briefly, a drop of blood from the infected mouse was added to $20 \mu \mathrm{l}$ of PLB $(1 \times$ Passive Lysis Buffer; Promega, Cat. No. E1941) contained in one well of a black, flat-bottom, 96-well plate (Greiner BioOne, Cat. No. 655900). As a negative control, blood was collected from a non-infected mouse. A 1:50 dilution of the NanoGlo substrate was prepared in NanoGlo ${ }^{\mathrm{TM}}$ luciferase assay buffer (Promega, Cat. No. N1120), $50 \mu \mathrm{l}$ of the dilution were added to the sample and the luminescence signal was read within $5 \mathrm{~min}$.

\section{Genotyping of transfected parasites}

Genomic DNA of transfected parasites was purified. Integration PCR was performed according to the schematic in Fig. 6. DNA sequences of primers used are shown in Table 1.

\section{Table 1 Primers used for genotyping in Fig. 6}

\begin{tabular}{ll}
\hline Primer & Sequence $\left(\mathbf{5}^{\prime} \mathbf{-} \mathbf{3}^{\prime}\right)$ \\
\hline 1 & GTGTAGTAACATCAGTTATTGTGTG \\
2 & ATACTGTATAACAGGTAAGCTGTTATTGTG \\
3 & TTTCCCAGTCACGACGTTG \\
4 & CTTAGTGTTTTGTATTAATGTCGATTTG \\
QCR2 & AGGGGCAAATACCAAAGTTGTT \\
QCR1 & ACGCATATTCACGAGTTTCACA \\
GW2 & CTTTGGTGACAGATACTAC \\
\hline
\end{tabular}

\section{Results}

This work presents an established protocol that allows efficient transfection of liver stage-derived merozoites in so-called detached cells and merosomes (Fig. 1). This protocol is based on the fact that in vitro, $P$. berghei-infected cells detach from their neighbouring cells and float into the cell culture supernatant. Thus, they can be easily purified by harvesting and then centrifuging the supernatant. After initially succeeding in transfecting detached cells/ merosomes, the next aim was to optimize the formation and yield of detached cells to quantify the corresponding number of merozoites used, and to standardize the protocol in a user-friendly way. As a first step, the optimal number of sporozoites to be used for host cell infection to maximize the generation of detached cells was determined (Fig. 2). As expected, with a constant number of host cells, the number of detached cells increases as the number of sporozoites used per infection increases. This increase is not linear and reaches a plateau with about 50,000 sporozoites. On average, using 50,000 sporozoites, about 550 detached cells were obtained when harvesting at $65 \mathrm{hpi} \mathrm{(Fig.} \mathrm{2a),} \mathrm{which} \mathrm{is} \mathrm{about} 20 \%$ of the parasites that were present at $48 \mathrm{hpi}$ (Fig. 2b). When higher sporozoite numbers were used, the number of detached cells formed also increased but the conversion rate of parasites present at 48 hpi to detached cells dropped considerably (Fig. 2b), which suggests sub-optimal parasite development.

Having established the optimal sporozoite number to use for infection, the effect of age of the parasite in the mosquito on the number of detached cells/merosomes formed and the corresponding merozoite number was determined (Fig. 3a). Detached cell formation was quantified, using sporozoites obtained from mosquitoes from day 16 to day 30 post-blood feed. Based on previous experiments, it was found that the success rate and efficiency of detached cell transfection starts to drop considerably when using fewer than 500 detached cells/ merosomes $\left(4 \times 10^{6}\right.$ merozoites). The minimum was set to 500 detached cells/merosomes and beyond day 24 postblood feed, numbers of detached cells formed dropped below this critical level. Accordingly, harvesting sporozoites from mosquitoes between day 16 and day 24 postblood feed results in the sufficient formation of detached cells. Thus, sporozoites can be used over a period of nine consecutive days, during which transfections can be flexibly and robustly planned and performed, as the processes from seeding of cells to liver stage-derived merozoite transfection takes place over a period of only 4 days.

For detached cell quantification, in addition to a fluorescence microscopy-based assay, a luminescence-based assay was adapted to determine the numbers of detached cells and merozoites (Fig. 3). Using this assay, it was 

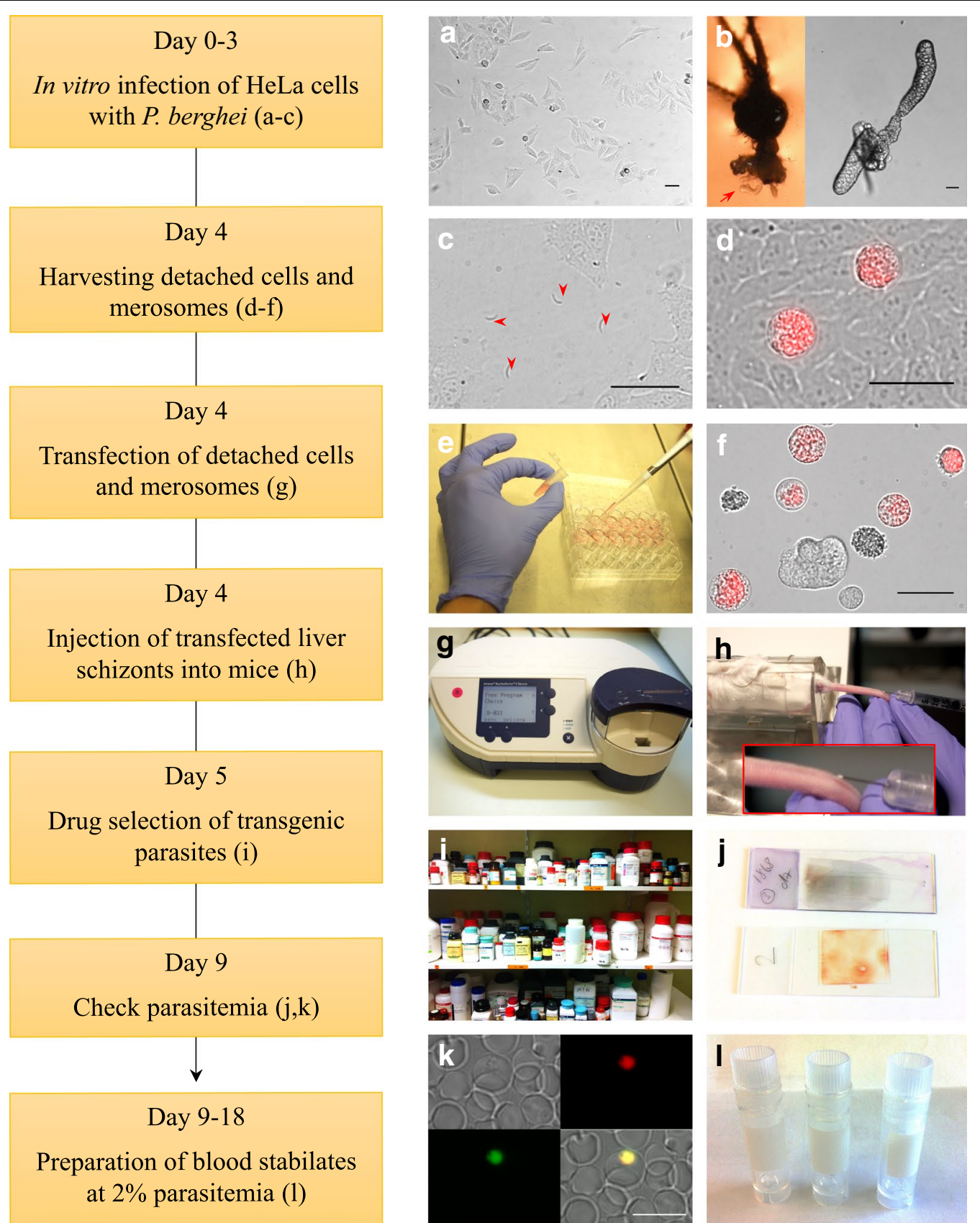

Fig. 1 Workflow and visualization of critical protocol steps. a Optimal density of seeded HeLa cells $24 \mathrm{~h}$ post seeding. b Representative images of dissection of mosquito salivary glands (red arrow) attached to the head (left) or removed (right). c HeLa cells infected with P. berghei sporozoites at a density representing sporozoite numbers used in the here described protocol. d HeLa cells infected with PbmCherry Hsp70 $_{0} 65$ hpi, prior to detached

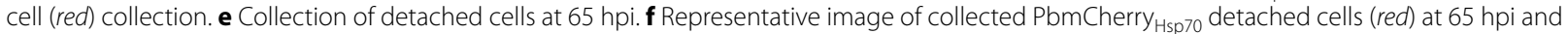
uninfected HeLa cell debris (unlabelled). $\mathbf{g}$ Amax ${ }^{\circledR}{ }^{\circledR}$ Nucleofector ${ }^{\circledR}$ II device with transfection program U33. h Intravenous injection of transfected parasites into a naïve mouse. i Drug selection of transgenic parasites. $\mathbf{j}$ Checking the parasitaemia by Wright stain or fluorescence. $\mathbf{k}$ Monitor transgenic parasites by fluorescence. I Preparation of blood stabilates. Scale bars in all images are $50 \mu \mathrm{m}$

calculated that about $4.5-8.5 \times 10^{6}$ merozoites are used per transfection (Fig. 3a).

However, experiments also showed that not only the quantity but also the viability and quality of detached cells and contained merozoites is key to successful transfection. Microscopic assessment is the fastest and most efficient way to assess detached cell morphology (Fig. 4). Transfection of detached cells had a better efficiency 


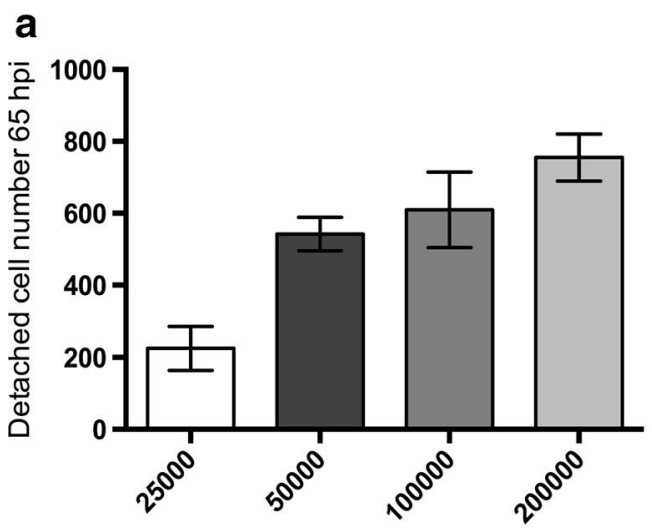

Number of sporozoites b

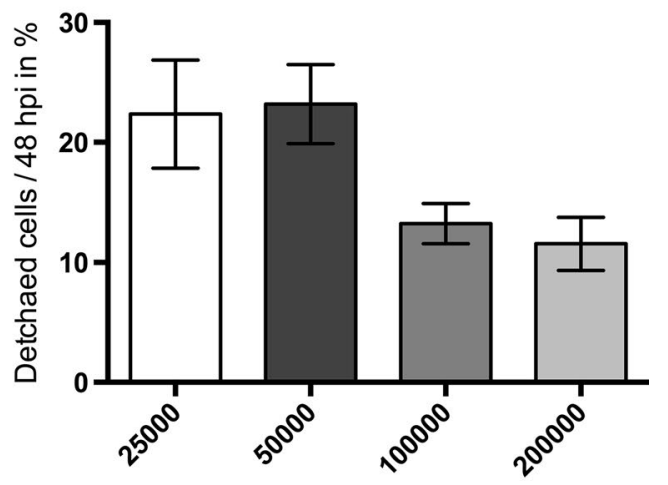

Number of sporozoites

Fig. 2 Experimental optimization of sporozoite numbers for HeLa cell infection to obtain maximal numbers of detached cells/merosomes. $\mathbf{a}$, $\mathbf{b}$

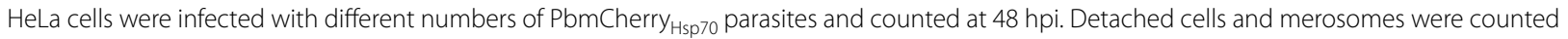
in the supernatant at $65 \mathrm{hpi}$. a Total number of detached cells formed at 65 hpi depending on the number of sporozoites used for infection. $\mathbf{b}$ Percentage of detached cells formed in relation to parasite count at 48 hpi was calculated. Shown are means with errors depicted as $95 \%$ confidence intervals, $n=3$ in biological triplicates

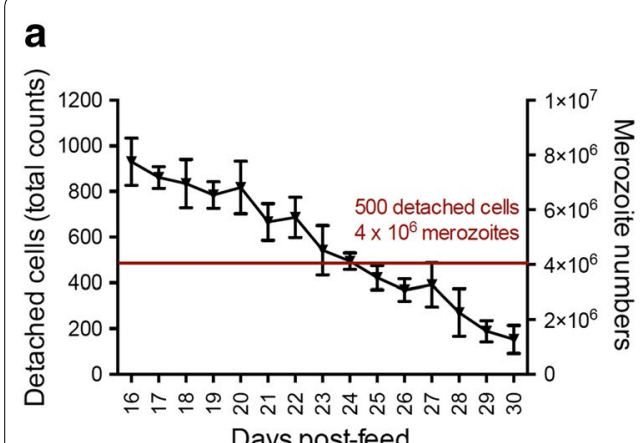

Days post-feed b

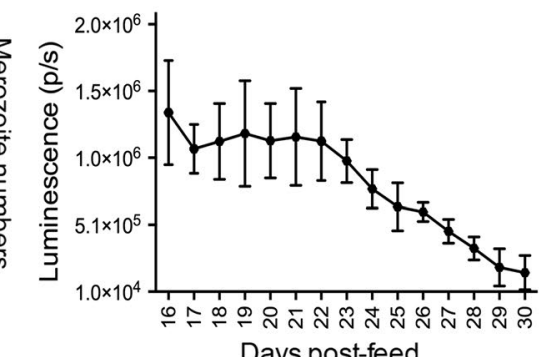

Days post-feed
C

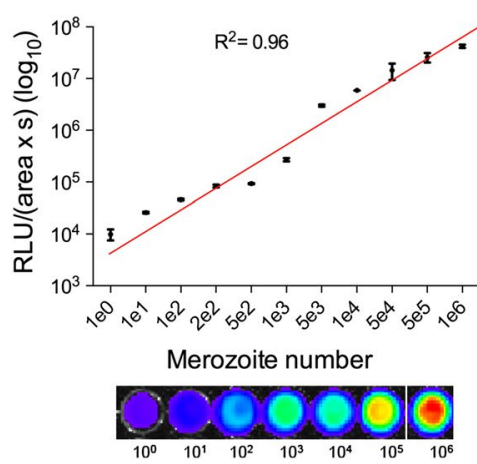

Fig. 3 Microscopic and luminescence-based determination of detached cell and merozoite number, dependent on the parasite age in infected mosquitoes. $\mathbf{a}$ The relation of the parasite age in infected mosquitoes to the number of detached cells obtained and the relative merozoite number was investigated from day 16 to day 30. A critical cut off (red line), in detached cell numbers obtained is at 500 detached cells $\left(4 \times 10^{6}\right.$ merozoites), as the success rate of detached cell transfections using fewer than 500 detached cells decreases. Seeding and infections were performed according to the present protocol, collected detached cells were counted using a fluorescent microscope at $65 \mathrm{hpi}$ and merozoite number was determined using a luminescence based assay, $n=3$. Error bars denote SD. $\mathbf{b}$ Determination of luminescence of detached cells obtained over time, using sporozoites from day 16 to day 30 post-blood feed. Detached cells of PbmCherry ${ }_{\mathrm{Hsp} 70}$-NLuc parasites were obtained and collected according to the present protocol. Aside from quantification of detached cell numbers by fluorescence, luminescence was used to estimate the number of detached cells used for transfection from day 16 onwards, following mosquito feeds. Luminescence correlates with number of detached cells contained, and suggests that at days 16-23, the number of viable merozoites remains relatively constant, while a significant decrease in luminescence is identified from day 24 onwards (denoting less detached cells and less merozoites). Values shown are the result of three independent experiments repeated in triplicate. Error bars denote SD. c PbmCherry $\mathrm{Hsp70}_{0}$-NLuc detached cells were collected and disrupted to release merozoites. The total number of merozoites of a single detached cell was counted, and a limiting dilution was performed to achieve one merozoite, which was lysed and its luminescence measured. The radiance calculated was $9.9 \times 10^{4} \mathrm{p} \mathrm{s}^{-1} \mathrm{~cm}^{-2}$. Performing a limiting dilution from 1 to $10^{6}$ merozoites we defined that a positive, significant $\left(R^{2}=0.96\right)$ correlation exists between merozoite number and the $\log _{10}$ of the relative light units of luminescence. This in turn allowed us to estimate the total number of merozoites in single individual detached cells and of the complete population of detached cells used for transfection. Values shown are the result of ten separate limiting dilutions from three independent experiments

when most of the detached cells contained viable and motile merozoites (Fig. 4a, b; Additional file 1: Movie S1) and only few abnormal detached cells were present
(Fig. 4c, d). Furthermore, it is also important to microscopically confirm that cells have not been contaminated during the process of infection with sporozoites, which 

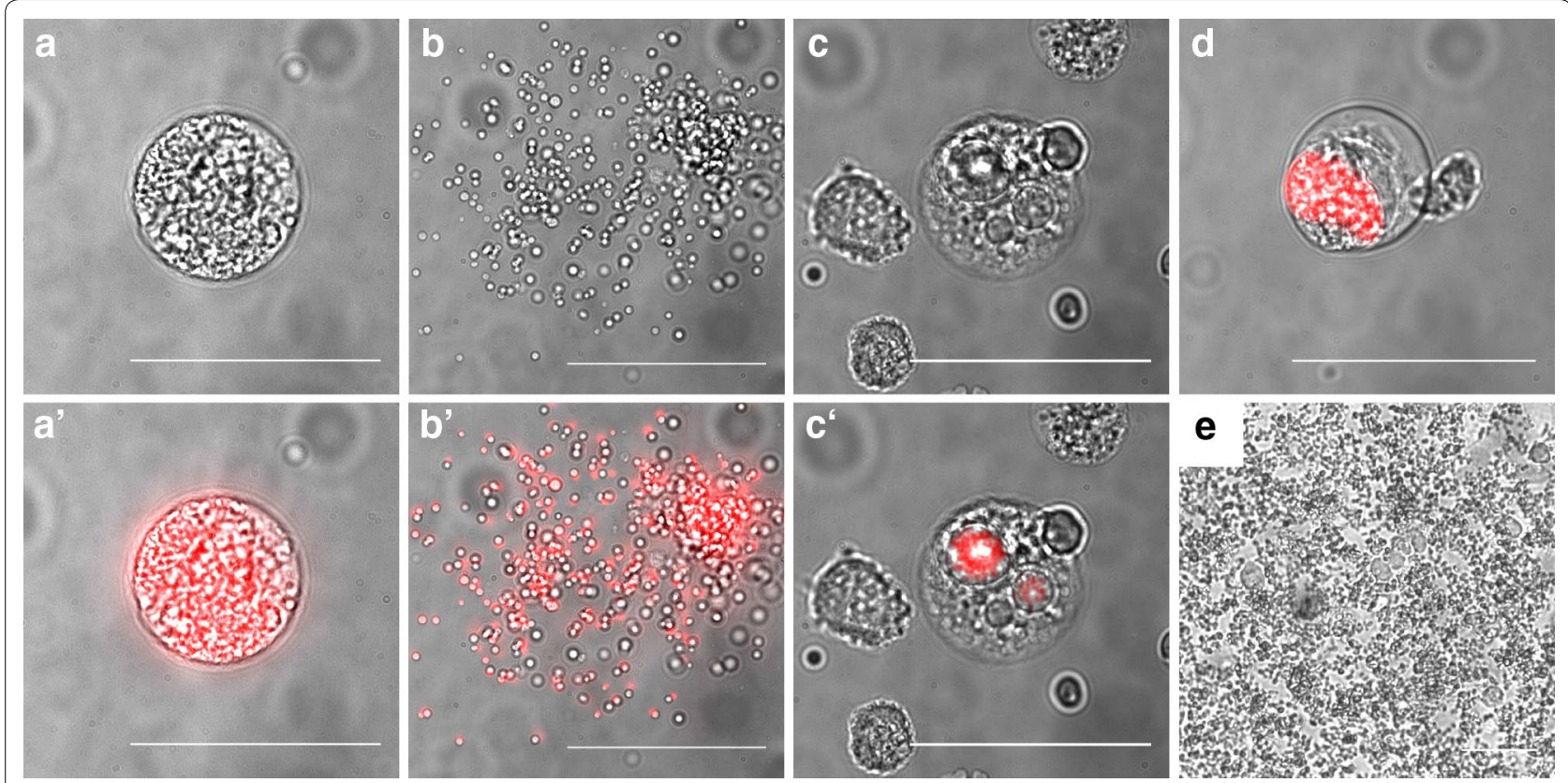

Fig. 4 Representative microscopic images of detached cells. a Bright field and $\mathbf{a}^{\prime}$ fluorescence merged image of a normal detached cell, $\mathbf{b}$ and $\mathbf{b}^{\prime}$ of a ruptured detached cell and $\mathbf{c}, \mathbf{d}$ examples of detached cells with abnormal morphology, $\mathbf{c}, \mathbf{c}^{\prime}$ of merofusosomes [25] and $\mathbf{d}$ of absent merozoite formation. example of a fungal contamination of Plasmodium infected cells. Scale bars are 50 um

can introduce bacteria and fungi, contained in the mosquito debris, to the cell culture (Fig. 3e). If contamination has occurred, transfection should not be performed, as contaminated detached cells must not be injected into mice.

This protocol was successfully used for transfection of DNA constructs facilitating integration into the parasite genome by single crossover homologous recombination (Figs. 5, 6; Table 2) to introduce DNA constructs for cytoplasmic GFP expression and expression of a luciferase reporter gene in addition to a previously introduced cytoplasmic mCherry expression cassette [18]. Moreover, DNA constructs facilitating integration into the parasite genome by double crossover homologous recombination, were also successfully transfected, for example for the generation of knock-out and complemented parasites (Figs. 5, 6; Table 2). DNA constructs were successfully transfected into different parasite backgrounds, such as wild type parasites, selection-marker-free cytoplasmic mCherry expressing parasites [20] and knock-out parasites. In conclusion, all techniques used to transfect blood stage parasites can also be used to manipulate liver stage-derived merozoites.

\section{Discussion}

The protocol described here allows the generation of transgenic $P$. berghei parasites. If infected mosquitoes are routinely accessible, the protocol offers a flexible experimental procedure and the potential to reduce the number of mice used per transfection. In addition to transfection of $P$. berghei blood stages, the new protocol provides an optimized usage of $P$. berghei parasites for transfection. Using liver stage-derived merozoites for transfection, mouse numbers used should be reduced most efficiently when the protocol is used for high numbers of transfections. Transfection of liver stage-derived schizonts and merozoites of either wild type P. berghei ANKA or transgenic selection-marker-free $P$. berghei ANKA parasites has been proven successful for transfection of DNA constructs facilitating integration into the parasite genome by both single- and double-crossover homologous recombination. Transfections performed with DNA constructs for integration by single homologous recombination had a slightly higher success rate than transfections performed with DNA constructs that integrated by double crossover homologous recombination. Most likely this is related to the mechanism of integration and whether or not expression can be episomal, rather than being a consequence of the transfection procedure itself $[23,24]$.

Transfection of liver stage-derived detached cells and merosomes should be successful for all genetic modifications that can be achieved by single or double crossover homologous recombination, such as expression of proteins fused with fluorescent, luminescent or other markers and interference with protein function by knock-out, 
a

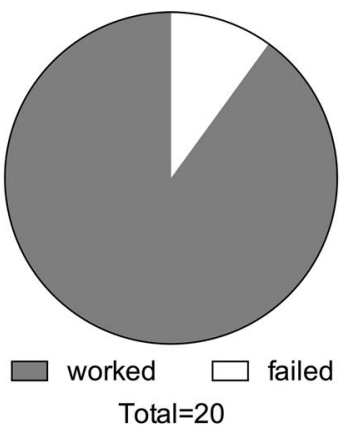

Success rate detached cell transfection b

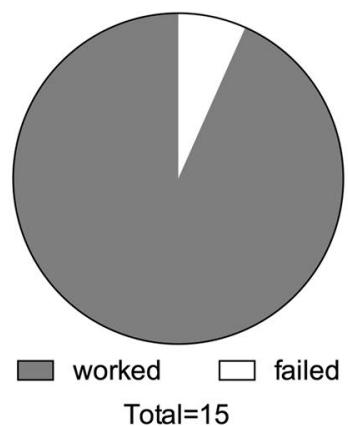

Integration via single crossover
C

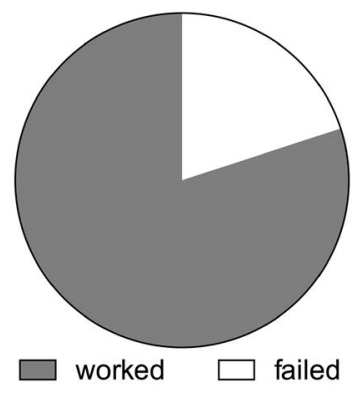

Total $=5$

Integration via double crossover
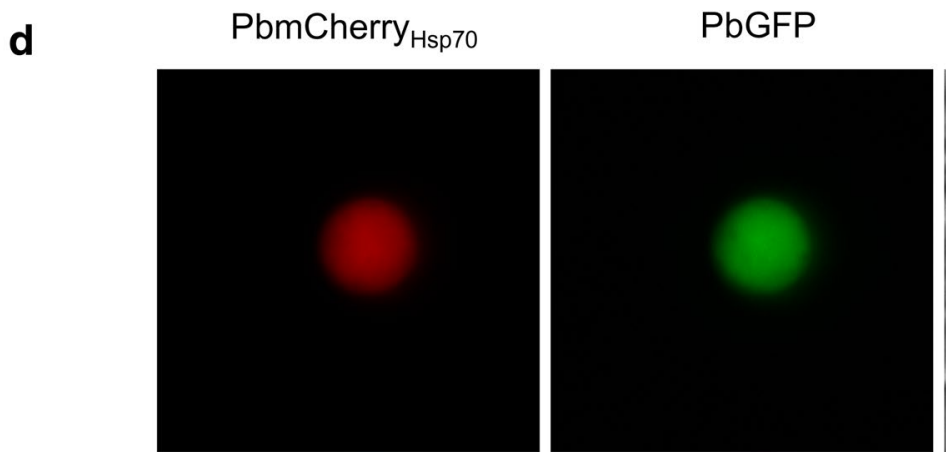

merge with DIC

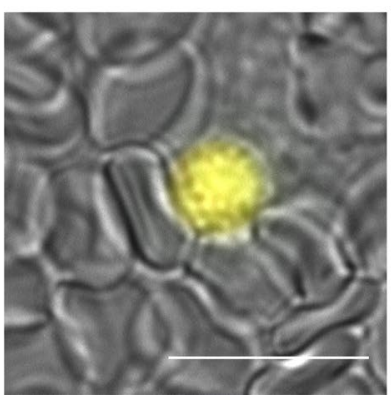

Fig. 5 Success rate of detached cell transfection of Plasmodium berghei parasites. Detached cells and merosomes of wild type or PbmCherry Hsp70 parasites were transfected according to the present protocol. a Illustration of the total success rate of detached cell transfection independent of parasites used and constructs transfected. b Success rate of detached cell transfection using DNA constructs allowing integration via either single

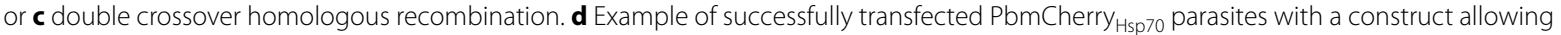
expression of cytoplasmic GFP. Scale bar is $10 \mu \mathrm{m}$

Table 2 shows the individual detached cell transfections performed that are summarized in Fig. 5a-c with information about the transfected construct, the parasite line that was transfected, the type of integration, the success rate, how often the transfection was performed and at which day after the transfection the mice became positive

\begin{tabular}{|c|c|c|c|c|c|}
\hline Construct & Parasite line & Integration & Success rate & $\begin{array}{l}\text { Number of } \\
\text { transfections }\end{array}$ & $\begin{array}{l}\text { Day mice } \\
\text { became positive }\end{array}$ \\
\hline PbSBP1KO (POB90-GFP) [19] & mCherry $_{\text {Hsp70 }}[20]$ & Double crossover & $50 \%(2)$ & 2 & 7 \\
\hline PbSBP1KO (POB90-GFP) [19] & mCherry $_{\mathrm{Hsp} 70} \mathrm{FLuc}_{\mathrm{ef1a}}[21]$ & Double crossover & $100 \%(1)$ & 2 & 6 \\
\hline PbSBP1KO (POB90-GFP) [19] & WT & Double crossover & $100 \%(1)$ & 1 & 7 \\
\hline PfSBP1 (PL0017-mCherry) [19] & PbSBP1KO [19] & Single crossover & $100 \%(1)$ & 1 & 8 \\
\hline PbGEM-336027 & mCherry $_{\mathrm{Hsp} 70}[20]$ & Double crossover & $100 \%(1)$ & 1 & 21 \\
\hline PbNLuc (PL0017) [18] & WT & Single crossover & $100 \%(1)$ & 1 & 5 \\
\hline PbNLuc (PL0017) [18] & mCherry $_{\text {Hsp70 }}[20]$ & Single crossover & $100 \%(2)$ & 2 & 4 \\
\hline PbNLuc (PL0017) [18] & mCherry $_{\mathrm{Hsp} 70} \mathrm{FLuc}_{\mathrm{ef1a}}[21]$ & Single crossover & $66.6 \%(3)$ & 3 & $4-5$ \\
\hline PL0017 GFP [22] & mCherry $_{\mathrm{Hsp} 70}[20]$ & Single crossover & $100 \%(8)$ & 8 & $5-12$ \\
\hline
\end{tabular}

conditional knock-out, modification, over-expression or replacement. In addition, this protocol should also be successful when introducing secondary genetic modifications such as complementation of previously knocked-out genes, additional marker fused proteins and double knock-outs.
A major advantage of the protocol is that liver stagederived merozoites can be collected and transfected in less than $20 \mathrm{~min}$, which is time saving and presumably beneficial for parasite viability. This is mainly because mature detached cells/merosomes can be directly 
a

Single crossover integration

c-ssu-rRNA locus
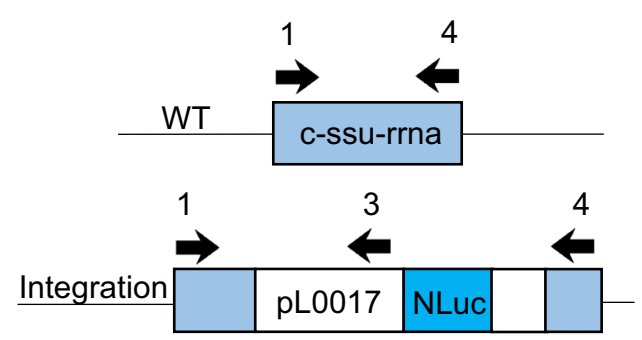

PbWT

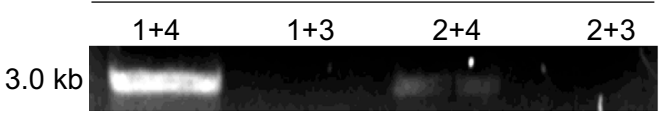

\section{d-ssu-rRNA locus}

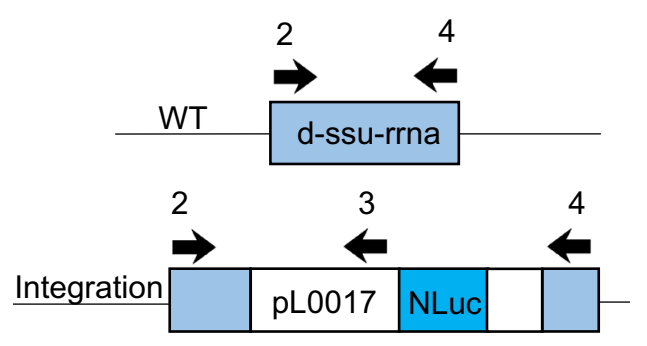

PbNLuc

\begin{tabular}{cccc}
\hline $1+4$ & $1+3$ & $2+4$ & $2+3$ \\
\hline & ntral & & \\
\hline
\end{tabular}

b

Double crossover integration

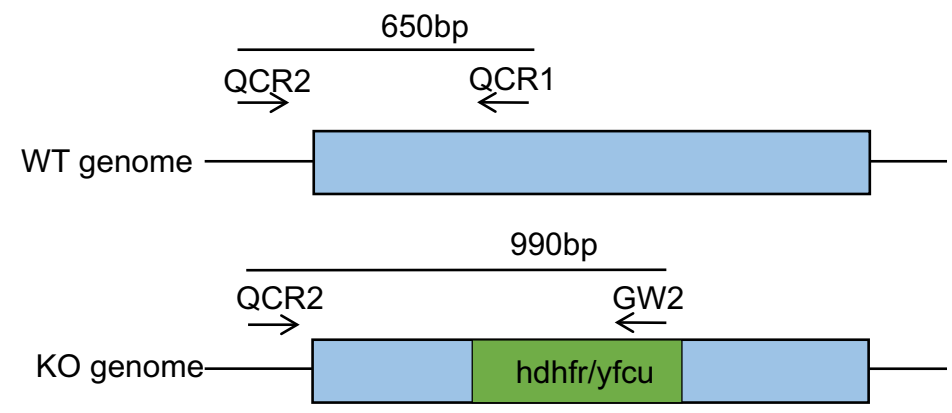

\begin{tabular}{|c|c|c|c|c|}
\hline & \multicolumn{2}{|c|}{ PCR: WT } & \multicolumn{2}{|c|}{ PCR: Integration } \\
\hline gDNA & PbWT & $\mathrm{PbKO}$ & PbWT & $\mathrm{PbKO}$ \\
\hline Primers & \multicolumn{2}{|c|}{ QCR2 + QCR1 } & \multicolumn{2}{|c|}{ QCR2 + GW2 } \\
\hline $990 \mathrm{bp}$ & & & & \\
\hline 650bp & & $=$ & & \\
\hline
\end{tabular}

Fig. 6 Integration PCR of parasite lines generated by detached cell transfection using a single or $\mathbf{b}$ double crossover integration. $\mathbf{a}$ P. berghei parasites were transfected with pL0017-NLuc [18], a construct that allows integration by single crossover into the P. berghei c-ssu-rRNA or $d$-ssu-rRNA locus. PCR amplification was used to verify integration of the plasmid into the $P$. berghei genome. The schematic shows the possible annealing regions of the primer pairs for the different parasite genotypes that could result. If the construct did not integrate into the parasite genome the primer pairs $1+4$ for the $c$-ssu-rRNA or $2+4$ for the $d$-ssu-rRNA locus generated a $3 \mathrm{~kb}$ PCR product. If the construct was successfully integrated, the primer pairs are too far apart ( $>14 \mathrm{~kb}$ ) to generate a PCR product. Primer pairs $1+3$ and $2+3$ are designed to confirm integration, a PCR product of $3 \mathrm{~kb}$ is generated only if the construct has integrated. PCR results of the PbNLuc parasites show integration into the c-ssu-rRNA locus, as the band for primer pair $1+4$ is absent and the primer pair $1+3$ gives a $3 \mathrm{~kb}$ band, there is no integration into the $d$-ssu-rRNA locus, which shows the same PCR products as for the WT parasites. $\mathbf{b}$ P. berghei parasites were transfected with PbGEM-336027, the construct facilitates integration of the hdhfr/ yfcu cassette via double crossover into the PBANKA_1145300 gene locus, resulting in a knock-out (KO). The primer pair QCR2 + QCR1 results in a 650 bp fragment, if parasites have a PBANKA_1145300 WT genotype. The primer pair QCR2 + GW2 is designed to show integration. A 990 bp PCR product is generated only, if the construct has integrated at the correct site of the parasite genome. The PCR results show integration of the hdhfr/ yfcu cassette into the PBANKA_1145300 gene locus. Transfected parasites are present in a mixed population of parasites having a PBANKA_1145300 KO genotype and PBANKA_1145300 WT genotype 
collected from the cell culture supernatant and need no further purification by density gradient centrifugation as is needed for the purification of blood stage schizonts.

The standard protocol for blood stage transfection published by Janse et al. recommends using a minimum of $10^{6}$ schizonts, corresponding to about $8-18 \times 10^{6}$ single merozoites per transfection [8]. Using the heredescribed protocol for transfection of liver stage-derived merozoites, about 4.5-8.5 $\times 10^{6}$ merozoites are used per transfection, depending on the number of detached cells obtained. An important observation was that efficient parasite development leading to the formation of detached cells is highly dependent on the age of parasites in infected mosquitoes. Therefore, it is recommended that mosquitoes are used between days 16 and 24 after blood feeding. Being able to use infected mosquitoes for 9 consecutive days to obtain detached cells, parasite transfections can be performed more flexibly in terms of timing and can be planned more robustly than is possible when performing blood stage transfections, which greatly depend on the parasitaemia of infected mice to be used for blood stage cultures. The liver stage development in vitro is independent of host circadian rhythms and accordingly, infections to obtain liver stage derived merozoites can be performed at any time of the day, offering flexible transfection conditions.

To obtain sporozoites needed for liver stage cultivation, a total of two mice is required: a pre-infection mouse in which infection is initially established from a blood stage stabilate of the parent $P$. berghei line, and a phenylhydrazine-treated recipient mouse (referred to as 'feed mouse'), which will be infected with the donor's blood, and thereafter used to feed the mosquitoes. Since only low blood volumes are passaged from the pre-infection mouse into the feed mouse, several feed mice can be infected from a single pre-infection mouse, to increase the number of mosquitoes that can be infected and thus the number of sporozoites that can be generated. In fact, in laboratories that routinely perform mouse infections with $P$. berghei, an additional pre-infection mouse is not required further reducing the number of experimental animals needed for the transfection.

During the optimization process of the protocol it was established that about 50,000 sporozoites are needed to obtain sufficient numbers of liver stage-derived merozoites per transfection. On average this sporozoite number can be obtained from three infected mosquitoes [18]. Normally, about 100-120 mosquitoes are fed on a single feed mouse. With a routine infection rate of about $70-80 \%$, about 80 infected mosquitoes were obtained. Per transfection, three mosquitoes that on average harbour about 50,000 sporozoites are dissected and used for the infection of HeLa cells. This infection results in about 500-1000 detached cells, which is sufficient for one transfection. Accordingly, it is possible to perform about 27 transfections with the number of infected mosquitoes that result from sacrificing a single feed mouse. Conversely, with the standard protocol used for transfection of blood stage-derived schizonts [8], which in theory requires 1 mouse per 4-5 transfections, performance of the same amount of transfections would require five mice.

Therefore, the protocol presented here is highly beneficial when intending to perform transfections in large scale. Transfection of liver stage-derived merozoites and schizonts has the potential to reduce the mouse usage by about fivefold in laboratories that have routine access to infected mosquitoes compared to the current protocol based on blood stage transfection. Since the in vitro liver stage development of Plasmodium takes place in cell culture plates, it is very suitable for scaling up the experimental design. Potential critical points could be that sporozoites, following isolation, should be used sufficiently quickly to ensure efficient host cell infection. Later in the experimental procedure, the time between detached cell purification and transfection is critical for parasite survival. Therefore, it is proposed that host cell infection with sporozoites and transfection of liver stage-derived parasites should be performed in sets, with a maximum of five transfections per set. Nevertheless, multiple infections could be prepared at time intervals if many transfections are planned.

\section{Conclusions}

So far, only one stage of Plasmodium berghei parasites, blood stage schizonts, could be transfected. Here a transfection protocol is described using liver stage-derived merozoites of $P$. berghei, which can be generated in vitro and in contrast to transfection of blood stage schizonts do not require sophisticated purification steps. The protocol described here provides more flexibility in terms of when and how many transfections can be performed. Moreover, transfection of liver stage-derived merozoites has the potential to result in a considerable reduction of mice used, if used by a lab that routinely has access to infected mosquitoes.

\section{Additional file}

Additional file 1: Movie S1. Motile merozoites within detached cells. Detached cells containing marker-free PbmCherry $_{\mathrm{Hsp} 70}$ parasites (red) were harvested at $65 \mathrm{hpi}$ and analysed using a fluorescence microscope. Images were acquired every $5 \mathrm{~s}$. Scale bar is $10 \mu \mathrm{m}$.

\section{Abbreviations}

DNA: deoxyribonucleic acid; GFP: green-fluorescent protein; hpi: hours post infection; PABA: para-aminobenzoic acid; PM: plasma membrane; PV: parasitophorous vacuole; PBS: phosphate-buffered saline. 


\section{Authors' contributions}

GK, RRS, MDN, PCB, and VTH conceived and designed the experiments. GK MDN, PCB, and LN performed experiments. GK and MDN analysed the data. GK, MDN and VTH drafted the manuscript. All authors read and approved the final manuscript.

\section{Author details}

${ }^{1}$ Institute of Cell Biology, University of Bern, Bern, Switzerland. ${ }^{2}$ Graduate School for Cellular and Biomedical Sciences, University of Bern, Bern, Switzerland. ${ }^{3}$ Present Address: Wellcome Centre for Molecular Parasitology, University of Glasgow, Glasgow, UK. ${ }^{4}$ Bernhard Nocht Institute for Tropical Medicine, Hamburg, Germany

\section{Acknowledgements}

We thank Chris J. Janse and Shahid M Khan for providing the selection-markerfree mCherry and the mCherryLuc expressing parasites. We thank Tobias Spielmann for providing the PfSBP1 and PbSBP1KO constructs. Images were acquired on equipment supported by the Microscopy Imaging Centre of the University of Bern. Financial support came from the Swiss National Foundation (310030_140691/1) (VTH) and the EVIMalaR EU network (FP7/2007-2013) (MDN and VTH).

\section{Competing interests}

The authors declare that they have no competing interests.

\section{Publisher's Note}

Springer Nature remains neutral with regard to jurisdictional claims in published maps and institutional affiliations.

Received: 23 February 2017 Accepted: 20 July 2017

Published online: 01 August 2017

\section{References}

1. Janse CJ, Franke-Fayard B, Mair GR, Ramesar J, Thiel C, Engelmann S, et al. High efficiency transfection of Plasmodium berghei facilitates novel selection procedures. Mol Biochem Parasitol. 2006;145:60-70.

2. Crabb BS, Rug M, Gilberger T-W, Thompson JK, Triglia T, Maier AG, et al. Transfection of the human malaria parasite Plasmodium falciparum. Methods Mol Biol. 2004;270:263-76.

3. Mota MM, Thathy V, Nussenzweig RS, Nussenzweig V. Gene targeting in the rodent malaria parasite Plasmodium yoelii. Mol Biochem Parasitol. 2001;113:271-8.

4. Hall N. A comprehensive survey of the plasmodium life cycle by genomic, transcriptomic, and proteomic analyses. Science. 2005:307:82-6.

5. Gardner MJ, Hall N, Fung E, White O, Berriman M, Hyman RW, et al. Genome sequence of the human malaria parasite Plasmodium falciparum. Nature. 2002;419:498-511.

6. Carlton JM, Angiuoli SV, Suh BB, Kooij TW, Pertea M, Silva JC, et al. Genome sequence and comparative analysis of the model rodent malaria parasite Plasmodium yoelii yoelii. Nature. 2002;419:512-9.

7. Moon RW, Hall J, Rangkuti F, Ho YS, Almond N, Mitchell GH, et al. Adaptation of the genetically tractable malaria pathogen Plasmodium knowlesi to continuous culture in human erythrocytes. Proc Natl Acad Sci USA. 2013;110:531-6

8. Janse CJ, Ramesar J, Waters AP. High-efficiency transfection and drug selection of genetically transformed blood stages of the rodent malaria parasite Plasmodium berghei. Nat Protoc. 2006;1:346-56.

9. Pfander C, Anar B, Brochet M, Rayner JC, Billker O. Recombinationmediated genetic engineering of Plasmodium berghei DNA. In: Ménard R, editor. Malaria. Methods in molecular biology (Methods and protocols), vol. 923. Totowa, NJ: Humana Press; 2012. p. 127-38.
10. Ménard R. Malaria methods and protocols. In: Ménard R, editor. Life Sci. Totowa: Humana Press; 2013.

11. Pfander C, Anar B, Schwach F, Otto TD, Brochet M, Volkmann K, et al. A scalable pipeline for highly effective genetic modification of a malaria parasite. Nat Methods. 2011;8:1078-82.

12. Stanway RR, Witt T, Zobiak B, Aepfelbacher M, Heussler VT. GFP-targeting allows visualization of the apicoplast throughout the life cycle of live malaria parasites. Biol Cell. 2009;101:415-30.

13. Lacroix C, Giovannini D, Combe A, Bargieri DY, Späth S, Panchal D, et al. FLP/FRT-mediated conditional mutagenesis in pre-erythrocytic stages of Plasmodium berghei. Nat Protoc. 2011;6:1412-28.

14. Philip N, Waters AP. Conditional degradation of Plasmodium calcineurin reveals functions in parasite colonization of both host and vector. Cell Host Microbe. 2015;18:122-31.

15. Sturm A, Heussler V. Live and let die: manipulation of host hepatocytes by exoerythrocytic Plasmodium parasites. Med Microbiol Immunol. 2007;196:127-33.

16. Sturm A, Amino R, van de Sand C, Regen T, Retzlaff S, Rennenberg A, et al. Manipulation of host hepatocytes by the malaria parasite for delivery into liver sinusoids. Science. 2006:313:1287-90.

17. Stanway R, Graewe S, Rennenberg A, Helm S, Heussler VT. Highly efficient subcloning of rodent malaria parasites by injection of single merosomes or detached cells. Nat Protoc. 2009;4:1433-9.

18. De Niz M, Stanway RR, Wacker R, Keller D, Heussler VT. An ultrasensitive NanoLuc-based luminescence system for monitoring Plasmodium berghei throughout its life cycle. Malar J. 2016;15:232.

19. De Niz M, Ullrich A, Heiber A, Soares AB, Pick C, Lyck R, et al. Virulence is conserved between rodent and human malaria parasites. Nat Commun. 2016;7:1-12.

20. Burda P-C, Roelli MA, Schaffner M, Khan SM, Janse CJ, Heussler VT. A Plasmodium phospholipase is involved in disruption of the liver stage parasitophorous vacuole membrane. PLoS Pathog. 2015;11:e1004760.

21. Prado M, Eickel N, De Niz M, Heitmann A, Agop-Nersesian C, Wacker R, et al. Long-term live imaging reveals cytosolic immune responses of host hepatocytes against Plasmodium infection and parasite escape mechanisms. Autophagy. 2015;11:1561-79.

22. Franke-Fayard B, Trueman H, Ramesar J, Mendoza J, van der Keur M, van der Linden $\mathrm{R}$, et al. A Plasmodium berghei reference line that constitutively expresses GFP at a high level throughout the complete life cycle. Mol Biochem Parasitol. 2004;137:23-33.

23. Menard R, Janse C. Gene targeting in malaria parasites. Methods. 1997:13:148-57.

24. Van Spaendonk RML, Ramesar J, Van Wigcheren A, Eling W, Beetsma $A L$, Van Gemert GJ, et al. Functional equivalence of structurally distinct ribosomes in the malaria parasite, Plasmodium berghei. J Biol Chem. 2001:276:22638-47.

25. Eickel N, Kaiser G, Prado M, Burda P-C, Roelli M, Stanway RR, et al. Features of autophagic cell death in Plasmodium liver-stage parasites. Autophagy. 2013;9:568-80.

\section{Submit your next manuscript to BioMed Central and we will help you at every step:}

- We accept pre-submission inquiries

- Our selector tool helps you to find the most relevant journal

- We provide round the clock customer support

- Convenient online submission

- Thorough peer review

- Inclusion in PubMed and all major indexing services

- Maximum visibility for your research

Submit your manuscript at www.biomedcentral.com/submit
Ciomed Central 